\title{
Long-term protection and mechanism of pacing-induced postconditioning in the heart
}

\author{
Fawzi A. Babiker • Ilka Lorenzen-Schmidt • Eric Mokelke • \\ Ward Y. Vanagt $\cdot$ Tammo Delhaas · Johannes Waltenberger • \\ Jack P. Cleutjens $\cdot$ Frits W. Prinzen
}

Received: 7 December 2009/Revised: 18 February 2010/Accepted: 13 March 2010/Published online: 25 March 2010

(C) The Author(s) 2010. This article is published with open access at Springerlink.com

\begin{abstract}
Brief periods of ventricular pacing during the early reperfusion phase (pacing-induced postconditioning, PPC) have been shown to reduce infarct size as measured after $2 \mathrm{~h}$ of reperfusion. In this study, we investigated (1) whether PPC leads to maintained reduction in infarct size, (2) whether abnormal mechanical load due to asynchronous activation is the trigger for PPC and (3) the signaling pathways that are involved in PPC. Rabbit hearts were subjected to $30 \mathrm{~min}$ of coronary occlusion in vivo, followed by 6 weeks of reperfusion. PPC consisted of ten 30-s intervals of left ventricular (LV) pacing, starting at
\end{abstract}

F. A. Babiker - I. Lorenzen-Schmidt · W. Y. Vanagt .

T. Delhaas · F. W. Prinzen $(\bowtie)$

Department of Physiology, Cardiovascular Research Institute

Maastricht, P. O. Box 616,

6200 MD Maastricht, The Netherlands

e-mail: Frits.Prinzen@FYS.unimaas.nl

W. Y. Vanagt · T. Delhaas

Division of Pediatric Cardiology, Department of Pediatrics, Cardiovascular Research Institute Maastricht,

Maastricht, The Netherlands

J. Waltenberger

Department of Cardiology,

Cardiovascular Research Institute Maastricht, Maastricht,

The Netherlands

J. P. Cleutjens

Department of Pathology,

Cardiovascular Research Institute Maastricht,

Maastricht, The Netherlands

E. Mokelke

Boston Scientific Corp., St. Paul, USA

F. A. Babiker

Department of Physiology, Faculty of Medicine,

Kuwait University, Kuwait, Kuwait reperfusion. PPC reduced infarct size (TTC staining) normalized to area at risk, from $49.0 \pm 3.3 \%$ in control to $22.9 \pm 5.7 \%$ in PPC rabbits. In isolated ejecting rabbit hearts, replacing LV pacing by biventricular pacing abolished the protective effect of PPC, whereas ten 30-s periods of high preload provided a protective effect similar to PPC. The protective effect of PPC was neither affected by the adenosine receptor blocker 8-SPT nor by the angiotensin II receptor blocker candesartan, but was abrogated by the cytoskeletal microtubule-disrupting agent colchicine. Blockers of the mitochondrial $\mathrm{K}_{\mathrm{ATP}}$ channel (5HD), PKC (chelerythrine) and PI3-kinase (wortmannin) all abrogated the protection provided by PPC. In the in situ pig heart, PPC reduced infarct size from $35 \pm 4$ to $16 \pm 12 \%$, a protection which was abolished by the stretch-activated channel blocker gadolinium. No infarct size reduction was achieved if PPC application was delayed by $5 \mathrm{~min}$ or if only five pacing cycles were used. The present study indicates that (1) PPC permanently reduces myocardial injury, (2) abnormal mechanical loading is a more likely trigger for PPC than electrical stimulation or G-coupled receptor stimulation and (3) PPC may share downstream pathways with other modes of cardioprotection.

Keywords Myocardial infarction - Postconditioning · Pacing $\cdot$ Cardioprotection $\cdot$ Mechanical load

\section{Introduction}

During the last 5 years, it has become evident in animals [51] as well as patients [34, 37] that interventions during the early reperfusion phase (postconditioning) can reduce the injury induced by the combination of ischemia and reperfusion. The conventional way to induce 
postconditioning is using repeated coronary occlusions after onset of reperfusion (ischemic postconditioning, IPoC). This method of protection has been shown to be triggered primarily by activation of adenosine receptors, to be mediated via $G_{i}$-coupled receptors (for review see [41]) and ultimately to lead to prevention of the opening of the mitochondrial permeability transition pore (mPTP) [1]. Several drugs can also induce postconditioning through $\mathrm{G}_{\mathrm{i}}$-coupled receptors, like bradykinin, anesthetics and opioids [41], whereas cyclosporine induces postconditioning by directly inhibiting the mPTP [1].

Our laboratory recently demonstrated that brief periods of ventricular pacing at essentially unchanged heart rate can also elicit myocardial protection during the early reperfusion phase [39]. This pacing-induced postconditioning (PPC) was demonstrated in the isolated rabbit heart as well as in the in vivo pig heart. For potential future clinical use, it is important to know whether PPC not only reduces infarct size after a few hours of reperfusion, but also in the long term. This question is important because studies have shown that reperfusion injury continues to develop for several days [19, 25, 52].

Moreover, it is of utmost importance to understand the mechanisms of PPC. Previous animal studies showed that ventricular pacing prior to ischemia (preconditioning) and in the early reperfusion phase (postconditioning) did not change myocardial blood flow or lactate release. This suggested, but did not prove, that ischemia is not a trigger for pacing preconditioning and PPC [39, 40]. In addition, PPC was demonstrated in isolated hearts with constant preload, afterload and heart rate, excluding these factors as potential triggers [39]. This information indicates that PPC, like IPoC, provides protection through endogenous mechanisms, but using at least partially different pathways than IPoC. Better understanding of all pathways that potentially lead to protection of the heart may provide approaches for more effective therapy during the early reperfusion phase.

Therefore, the aims of the present study were to investigate the long-term protection by PPC as well as its mechanisms. With respect to the latter, we hypothesized that abnormal myocardial mechanical load, induced by the dyssynchronous contraction during pacing, is the trigger for PPC. Of special interest seems to be the early systolic stretch in regions remote from the pacing site (late-activated regions) [26, 27]. To investigate the importance of stretch as trigger for PPC, we compared the protective effect of single ventricular (dyssynchronous) and biventricular (relatively synchronous) pacing and that of increased global ventricular preload without pacing. Moreover, it was investigated to what extent the protective effect of PPC is abolished by a blocker of stretch-activated channels (SACs), disruption of microtubule stretch- mediated signaling and blockers of various receptors and signaling pathways, involved in other modes of postconditioning (adenosine, phosphatidylinositol-3 kinase (Pi3K), protein kinase $\mathrm{C}$ (PKC) and the mitochondrial $\mathrm{K}_{\mathrm{ATP}}$ channel (mito $\mathrm{K}_{\mathrm{ATP}}$ ). Studies were performed in rabbit as well as pig hearts because we previously showed the efficacy of PPC in both species [39].

\section{Materials and methods}

Rabbit heart experiments

Animal handling and treatment were according to the Dutch law on animal experimentation (WOD). The protocol was approved by the Animal Ethical Committee of Maastricht University. Female white New Zealand rabbits (SPF bred, Harlan, France) were used for all rabbit studies.

Long-term PPC studies in rabbits

Anesthesia was induced with ketamine (15 mg/kg, I.M.) and diazepam (0.5 mg/kg, I.M.) and maintained by I.V. infusion of sufentanyl $(0.5-2 \mu \mathrm{g} / \mathrm{kg} / \mathrm{min})$, diazepam (1 mg/ $\mathrm{kg} / 4 \mathrm{~h}$ ) and ventilation with isoflurane (0.5-1.0\%). After opening the chest, the left descending coronary artery was isolated and ligated using a 3-0 suture for an ischemic period of $30 \mathrm{~min}$. In the PPC group, PPC was applied starting $30 \mathrm{~s}$ after opening the vessel and applying ten $30-\mathrm{s}$ periods of pacing (at 30-s non-paced intervals) at the $\mathrm{LV}$ free wall, posterior from the previously ischemic region (VOO pacing mode, $\sim 10 \%$ above the sinus rhythm). The chest was closed after $1 \mathrm{~h}$ of reperfusion. In the control group, no other intervention than reperfusion was performed. All animals were allowed to recover after closing the chest, anesthesia was stopped and the animals were returned to their cages.

The effect of myocardial infarction and PPC on LV geometry was determined using 2D echocardiography before and every 2 weeks after myocardial infarction. To this purpose, short-axis views at mid-papillary level were recorded in the conscious state using an ESAOTE Megas ultrasound System and a 5-7.5 MHz transducer.

After 6 weeks, the animals were anesthetized again and hemodynamic measurements were performed using a $4 \mathrm{~F}$ Millar pressure transducer. All signals (ECG, pressure) were digitized at $1 \mathrm{kHz}$ and stored using custom-made software (IDEEQ, Maastricht Instruments, The Netherlands) and later analyzed using custom software.

Finally, the heart was arrested and infarct size was determined using methylene blue and triphenyl tetrazolium chloride (TTC) staining as previously described [39]. 
Isolated rabbit heart studies

After sedating the animals with intramuscular ketamine $(50 \mathrm{mg} / \mathrm{kg})$ and xylazine $(5 \mathrm{mg} / \mathrm{kg})$, hearts were isolated and attached to the perfusion apparatus as described in more detail by Vanagt et al. [40]. Briefly, the aorta and a pulmonary vein were cannulated to allow antegrade perfusion (ejecting mode) of the isolated heart. The heart was instrumented with epicardial pacing electrodes on the right atrial (RA) appendage and on the posterior basal left ventricular (LV) wall. In the case of biventricular (BiV) pacing, another pacing electrode was positioned on the right ventricular (RV) free wall. A silastic catheter to measure LV pressure was introduced into the LV through the LV apex. Throughout the experiment, the preload was kept constant at $6 \mathrm{mmHg}$ (except in the high preload group, see below), afterload at $80 \mathrm{mmHg}$ and heart rate at $240 \mathrm{bpm}$ (physiological heart rate for rabbits [3]).

All hearts experienced $30 \mathrm{~min}$ of coronary occlusion, followed by $2 \mathrm{~h}$ of reperfusion (Fig. 1). Hearts subjected to PPC additionally underwent ten cycles of $30 \mathrm{~s}$ ventricular pacing on/30 s ventricular pacing off (except when mentioned otherwise). Ventricular pacing was performed using simultaneous atrio-ventricular pacing (atrio-ventricular interval $=0 \mathrm{~ms}$ ) to insure complete ventricular activation. BiV pacing was achieved by simultaneous $\mathrm{LV}$ and $\mathrm{RV}$ pacing. In the high preload group, preload was increased from 6 to $12 \mathrm{mmHg}$ by raising the atrial reservoir in the working heart model using ten cycles of $30 \mathrm{~s}$ high preload and $30 \mathrm{~s}$ of normal preload.

In groups where drugs were administered to investigate involvement of their target in PPC, the hearts were perfused with drug containing medium between 5 min prior to onset of reperfusion and $10 \mathrm{~min}$ after start of reperfusion (the end of the PPC sequence, Fig. 1). Due to the nonrecirculating perfusion system used, drug levels in the perfusion medium can be expected to have decreased to zero within a few minutes.

All drugs, 8-(p-sulfophenyl)theophylline (8-SPT, $100 \mu \mathrm{mol} / \mathrm{l})$, candesartan $(10 \mathrm{nmol} / \mathrm{l}), 5$-hydroxydecanoate (5-HD, $100 \mu \mathrm{mol} / \mathrm{l})$, chelerythrine $(5 \mu \mathrm{mol} / \mathrm{l})$, wortmannin $(100 \mathrm{nmol} / \mathrm{l})$ and colchicine $(50 \mu \mathrm{mol} / \mathrm{l})$ were obtained from Sigma-Aldrich (St. Louis, Missouri). These drugs and the doses used have previously been shown to selectively block their target by several others [7, 24, 35, 45, 48].

Collection of hemodynamic data and infarct size determination (TTC staining) were similar to the ones used in our previous study [40].

In vivo swine hearts

Swine studies were performed in accordance with the National Institutes of Health Guidelines for the Care and
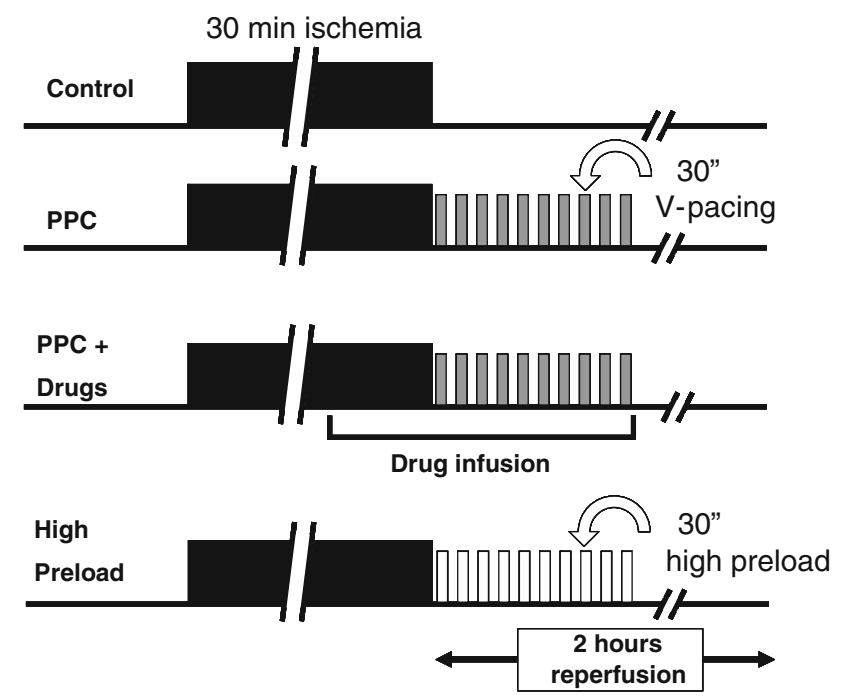

Fig. 1 Experimental protocol for studying the role of abnormal mechanical load and electrical stimulation in isolated rabbit hearts. Regional ischemia was induced by coronary ligation for $30 \mathrm{~min}$. The hearts were atrially paced at $240 \mathrm{bpm}$ during the entire protocol, except during the first 10 min of reperfusion (gray blocks), where simultaneous atrial and LV posterior wall (or biventricular in one series) pacing at $240 \mathrm{bpm}$ was performed. Drug infusion was started $5 \mathrm{~min}$ before the start of reperfusion and was continued till the end of LV pacing. The ten open blocks in the high preload group indicate the periods with increased preload (from 6 to $12 \mathrm{mmHg}$ ) by raising the atrial reservoir

Use of Laboratory Animals. The protocol was reviewed and approved by the Boston Scientific Institutional Animal Care and Usage Committee (Boston Scientific Corp., St. Paul, MN, USA). A total of 26 domestic female swine were randomly assigned to control $(\mathrm{C})$, pacing post-conditioning using ten 30-s cycles (PPC-10), five 30-s cycles (PPC-5), 5 min delayed PPC-10 (PPC-10D) and PPC with gadolinium $(\mathrm{PPC}+\mathrm{Gd})$. Group size was 5, except for the PPC-10 group $(n=6)$. Gadolinium is a potent inhibitor of stretchactivated channels in the myocardium $[4,9]$ and has been shown to block volume overload-induced cardioprotection in other animal models [8, 22].

The procedure was conducted in a closed chest model of balloon-induced myocardial infarction. A surgical plane of anesthesia was maintained with isoflurane (1.5-2.5\%). Two bi-polar endocardial pacing leads (Fineline II, Boston Scientific Corp, St. Paul, MN, USA) were introduced transvenously and positioned in the right atrium and RV apex. An 8 Fr introducer sheath (Super Sheath, Boston Scientific Corp, St. Paul, MN, USA) was surgically inserted into a femoral artery using the Seldinger technique. A 6-8 Fr guide catheter (Boston Scientific Corp, St. Paul, MN, USA) was advanced into the femoral artery and the ostium of the catheter was positioned near the ostium of the left main coronary artery. An initial angiogram was taken to measure the diameter of the left anterior descending artery (LAD) for determining the appropriate occlusion 
balloon size. The occlusion balloon (Maverick 2, Boston Scientific Corp, St. Paul, MN, USA) was positioned distal to the first branch of the LAD as deemed necessary for creating an infarct of appropriate size. After $30 \mathrm{~min}$ of a stabilization period, coronary artery occlusion was induced by inflation of the balloon catheter. The occlusion was confirmed by fluoroscopy performed several times during the occlusion period. After $60 \mathrm{~min}$ of occlusion, the balloon was rapidly deflated, followed by $3 \mathrm{~h}$ of reperfusion.

In all pacing protocols, right ventricular (RV) pacing was performed in the VVI mode (ventricular sense-ventricular pace, inhibit) $20-30$ beats above sinus rhythm (SR). In the control group, hearts were allowed to beat in SR throughout the experiment. In the PPC-10 and PPC-5 groups, postconditioning was initiated exactly $30 \mathrm{~s}$ after the deflation of the balloon, using ten or five cycles of $30 \mathrm{~s}$ of RV pacing followed by $30 \mathrm{~s}$ of normal SR, respectively (Fig. 2). In the delayed PPC (PPC-D) group, the pacing sequence was initiated 5 min after the balloon was deflated. In the PPC $+\mathrm{Gd}$ group, at the end of the index ischemia, $\mathrm{Gd}^{3+}(40 \mu \mathrm{mol})$ was injected into the left main artery via the guide catheter throughout the entire 10-min PPC period. In all groups, the reperfusion period was $3 \mathrm{~h}$, after which the animal was overdosed with isoflurane. Thereafter, methylene blue and TTC staining were performed as previously described [39]. Area of infarct, area at risk and total LV area were determined using ImageJ (NIH, Bethesda, MA, USA).

\section{Statistical analysis}

Data are expressed as mean $\pm \mathrm{SD}$. Unpaired two-sided $t$ tests were used to test for differences in infarct size
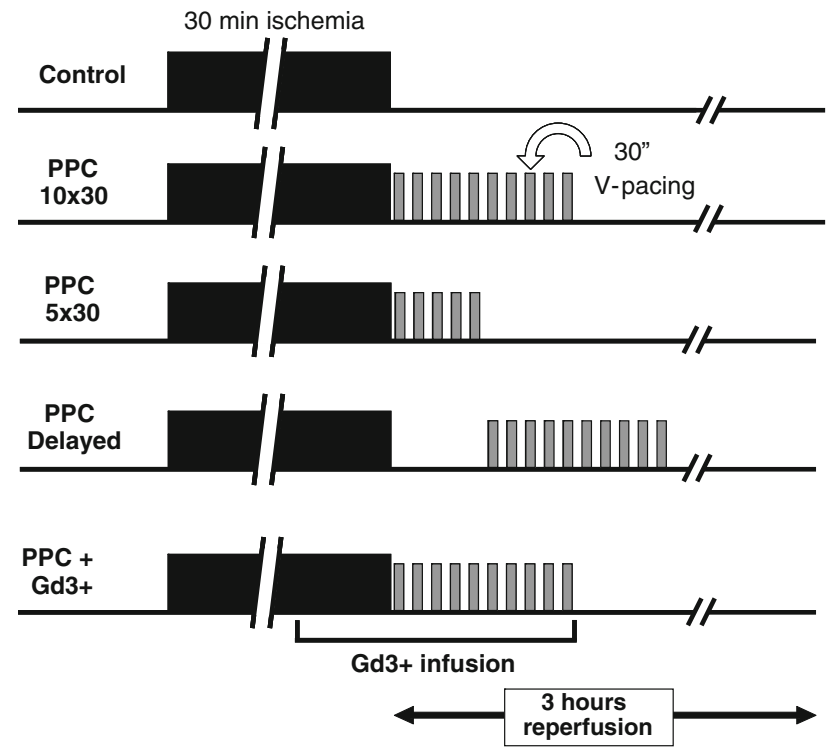

Fig. 2 Experimental protocol for the in vivo studies of pig hearts. The format is equal to that of Fig. 1 between groups. Repeated measures one-way analysis of variance (ANOVA), followed by Bonferroni post hoc tests, were used to evaluate hemodynamic changes over time. $P<0.05$ was considered to be statistically significant.

\section{Results}

Long-term PPC studies

Infarct size (relative to area at risk, AAR) was $49.0 \pm 8.6 \%$ in the controls $(n=5)$ and $22.9 \pm 15.0 \%$ in the PPC rabbits $(n=7),(P=0.006$, Fig. 3$)$. The area at risk was not significantly different at $41.6 \pm 6.9$ and $38.5 \pm 5.7 \%$ of the LV, respectively. Two weeks after the index ischemia, a significant decrease in fractional shortening was observed in the control group, but not in the PPC group. LV end-diastolic diameter showed a sustained significant increase in both groups during the weeks following the infarction, but this increase tended to be smaller in the PPC group (Fig. 4). Invasive hemodynamic measurements after 6 weeks of infarction did not show a significant difference between the groups with regard to heart rate, systolic and end-diastolic blood pressure and the maximal rate of rise of $\mathrm{LV}$ pressure, $\mathrm{LVd} P / \mathrm{d} t_{\max }$ (data not reported).

\section{Isolated rabbit hearts}

There was no significant difference in body weights and heart weights between the study groups. The area at risk of the LV was also not significantly different between any of the study groups (Table 1).

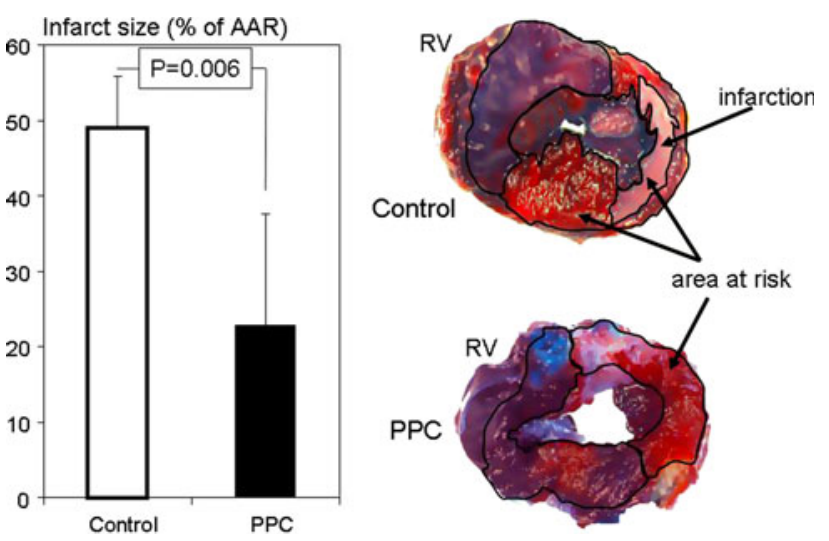

Fig. 3 Infarct size, normalized for the area at risk, in rabbit hearts 6 weeks after the ischemia/reperfusion procedure (left) and representative hearts (right). Open bar represents controls and filled bar represents PPC 

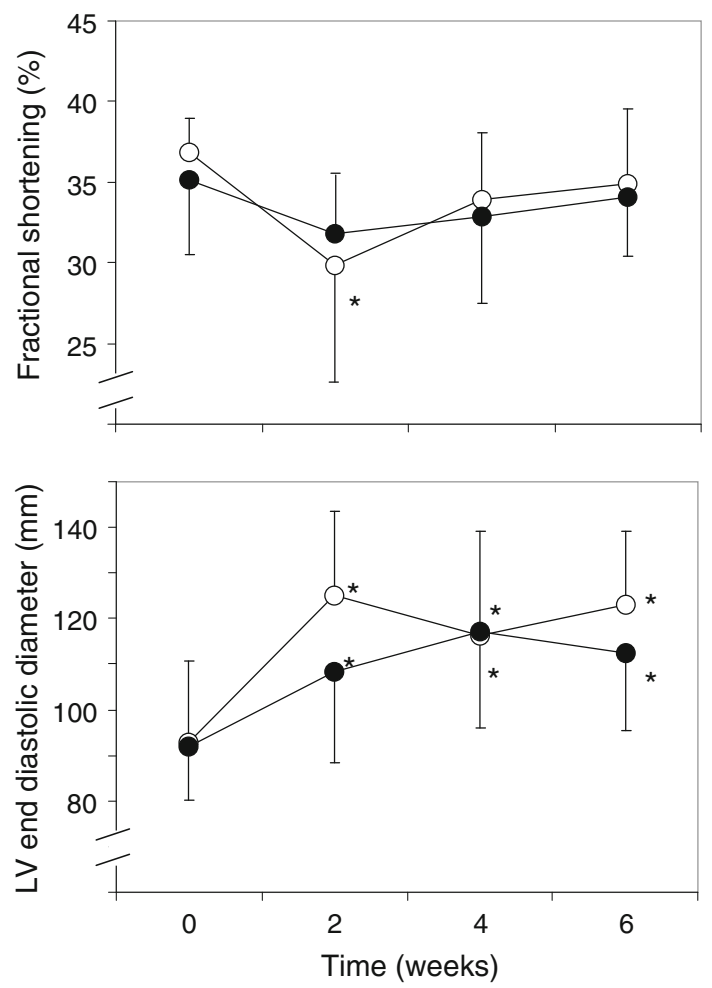

Fig. 4 Time course of fractional shortening and LV end-diastolic diameter during the 6-week follow-up in rabits (right). Open bar and open symbols represent controls and filled bar and symbols represent PPC. $* P<0.05$ as compared to time $=0$ (pre-ischemia)

Table 1 Number of experiments per group, body weight $(\mathrm{kg})$, heart weight, area at risk (\% of LV) and infarct size (\% of area at risk) in the various groups of isolated rabbit hearts

\begin{tabular}{llllll}
\hline Group & $n \begin{array}{l}\text { Body } \\
\text { weight }\end{array}$ & $\begin{array}{l}\text { Heart } \\
\text { weight }\end{array}$ & $\begin{array}{l}\text { Area at } \\
\text { risk }\end{array}$ & $\begin{array}{l}\text { Infarct } \\
\text { size }\end{array}$ \\
\hline Control & 9 & $3.02 \pm 0.61$ & $8.46 \pm 0.75$ & $54 \pm 6$ & $47 \pm 3$ \\
Paced & 8 & $3.03 \pm 0.35$ & $8.60 \pm 0.45$ & $51 \pm 6$ & $26 \pm 5^{*}$ \\
BiV pacing & 4 & $2.45 \pm 0.31$ & $7.61 \pm 0.53$ & $53 \pm 2$ & $51 \pm 2$ \\
Preload & 4 & $3.50 \pm 0.42$ & $8.20 \pm 0.63$ & $48 \pm 3$ & $23 \pm 2^{*}$ \\
8-SPT & 5 & $2.76 \pm 0.71$ & $8.46 \pm 1.35$ & $55 \pm 5$ & $25 \pm 7^{*}$ \\
Colchicine & 6 & $3.53 \pm 0.21$ & $8.73 \pm 0.61$ & $54 \pm 11$ & $53 \pm 2$ \\
Candesartan & 4 & $3.43 \pm 0.11$ & $8.45 \pm 0.51$ & $50 \pm 4$ & $20 \pm 2^{*}$ \\
5HD & 6 & $2.83 \pm 0.22$ & $8.57 \pm 0.44$ & $57 \pm 8$ & $49 \pm 5$ \\
Wortmannin & 4 & $2.93 \pm 0.43$ & $8.78 \pm 0.11$ & $55 \pm 9$ & $50 \pm 4$ \\
Chelerythrine & 4 & $2.84 \pm 0.20$ & $7.94 \pm 0.34$ & $52 \pm 8$ & $45 \pm 4$ \\
\hline
\end{tabular}

* $P<0.05$, as compared to control

\section{Hemodynamic changes}

At baseline during RA pacing, cardiac output and $\mathrm{LVd} P$ / $\mathrm{d} t_{\max }$ were similar in all groups. These variables were also not significantly different between the groups at any time point during the protocol (Table 2). In all groups, during and after ischemia, cardiac output and $\mathrm{LVd} P / \mathrm{d} t_{\max }$ were significantly depressed as compared with baseline. During early reperfusion in the PPC groups, LV pacing caused a moderate reduction in cardiac output and $\mathrm{LVd} P \mathrm{~d} t_{\max }$ as compared with RA pacing (Table 2). In the BiV PPC group, biventricular pacing did not lead to a significant decrease in hemodynamic parameters as compared to atrial pacing. In the high preload group, hemodynamic indices increased significantly during the episodes with high preload as compared to those with normal preload. None of the drugs had a significant effect on hemodynamics (Table 2).

\section{Infarct size reduction}

Similar to our previous study [39], single site pacing at the $\mathrm{LV}$ wall (PPC) resulted in a significant reduction in infarct size (26 \pm 5 vs. $47 \pm 3 \%$ in the control group). LV pacing in the early reperfusion phase was shown to lead to significant redistribution of mechanical load and abnormal patterns of shortening and stretch within the LV wall [27, 39]. In the high preload group, a higher workload in the absence of any ventricular pacing (evidenced by increased cardiac output at fixed afterload and heart rate) was associated with a similar protection (infarct size $23 \pm 2 \%$ ) as compared to PPC. In contrast, biventricular pacing, known to lead to much smaller regional contraction abnormalities than LV pacing [43], did not provide significant protection (infarct size $51 \pm 2 \%$, Fig. 5; Table 2).

Figure 5 also shows that the adenosine receptor blocker 8-SPT did not abolish the PPC-induced protection $(25 \pm 7 \%)$. These data indicate that this autocrine factor, released by the myocardium during ischemia, is not involved in PPC. In contrast, the microtubule-disrupting agent colchicine abolished the PPC-induced protection (53 $\pm 2 \%$, Fig. 5), suggesting a role for the cytoskeleton in PPC.

The blockers of PKC, PI3K and mitochondrial $\mathrm{K}_{\mathrm{ATP}}$ (chelerythrine, wortmannin and 5-HD, respectively) all abrogated the protective effect of PPC (45 $\pm 4,50 \pm 4$ and $49 \pm 5 \%$, respectively), thus providing evidence that these signaling processes, known to be involved in IPoC, are also involved in PPC (Fig. 5).

\section{Algorithm for PPC}

To investigate what combination of duration and number of pacing cycles provides protection, experiments were performed where, in addition to the $10 \times 30^{\prime \prime}$ pacing protocol, also $10 \times 20^{\prime \prime}$ and $10 \times 10^{\prime \prime}$ as well as $5 \times 30^{\prime \prime}, 5 \times 20^{\prime \prime}$ and $5 \times 10^{\prime \prime}$ pacing was performed. Figure 6 displays the infarct size achieved using the various pacing algorithms, plotted as a function of the total duration of pacing. Algorithms using ten cycles of ventricular pacing resulted 


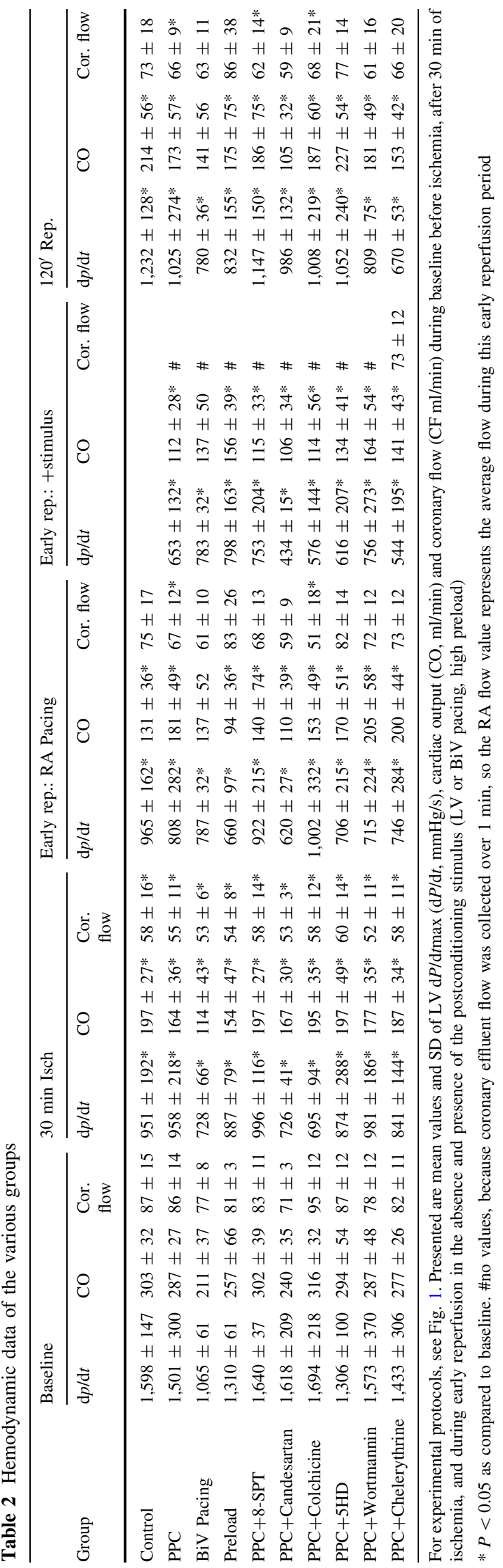

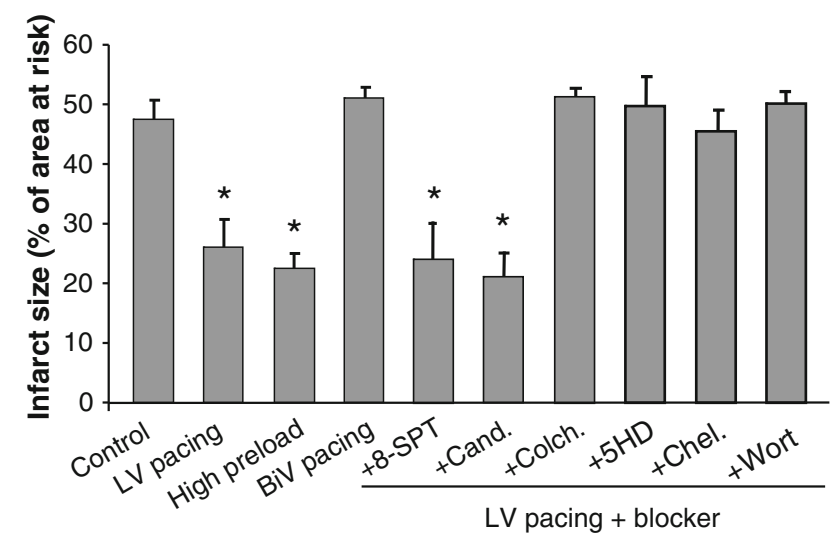

Fig. 5 Infarct size, normalized to the area at risk, in isolated rabbit hearts. Infarct size in the control group is compared with that in hearts subjected to ten cycles of $30 \mathrm{~s}$ of: left ventricular (LV) pacing, high preload and biventricular $(\mathrm{BiV})$ pacing as well as ten cycles of $30 \mathrm{~s} \mathrm{of}$ LV pacing plus administration of 8-SPT (adenosine receptor blocker, PPC+8-SPT), colchicine (disrupting microtubules, PPC+colchicine), $5 \mathrm{HD}$ (mitochondrial $\mathrm{K}_{\mathrm{ATP}}$ channel blocker, $\mathrm{PPC}+5 \mathrm{HD}$ ), chelerythine (PKC inhibitor, $\mathrm{PPC}+\mathrm{Chel}$ ) and wortmannin (PI3-kinase inhibitor, PPC + Wort). The drugs were added to the perfusion medium between 25 min of coronary occlusion and $10 \mathrm{~min}$ of reperfusion (see Fig. 1). Values are mean $\pm \mathrm{SD}$. ${ }^{*} P<0.05$, as compared to control. The number of animals is indicated in Table 1

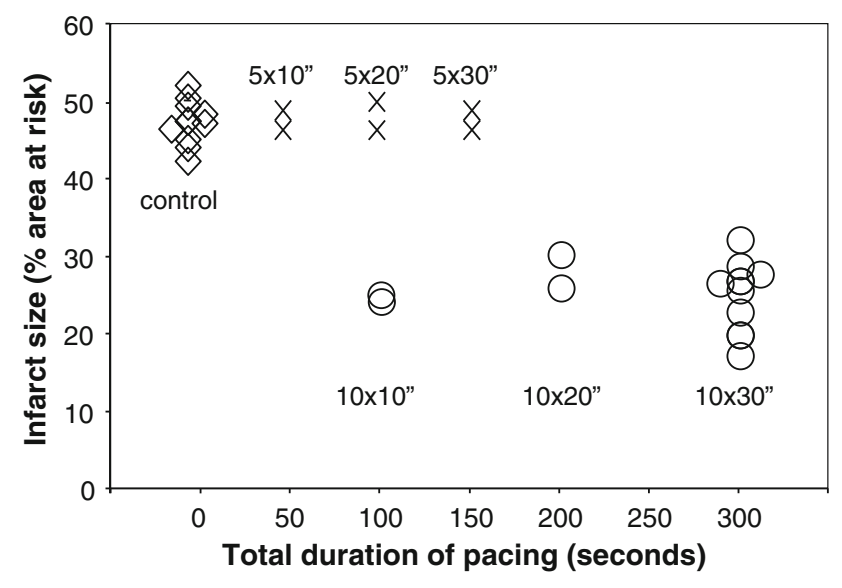

Fig. 6 Algorithm for pacing postconditioning. Infarct size (expressed as percentage of the area at risk) is plotted as a function of the total duration of pacing. Each symbol represents one rabbit heart

in protection (circles), but no algorithms using five cycles of pacing resulted in protection, not even if the total duration of pacing was longer than that of the $10 \times 10^{\prime \prime}$ pacing protocol (Fig. 6).

Pig heart studies

In the in situ pig hearts, PPC reduced infarct size from $\sim 35$ to $\sim 16 \%$ of the area at risk, a relative reduction similar to that in the rabbit hearts (Fig. 7). Applying only five instead of ten pacing cycles provided less, and statistically not significant, protection than ten cycles. Starting 


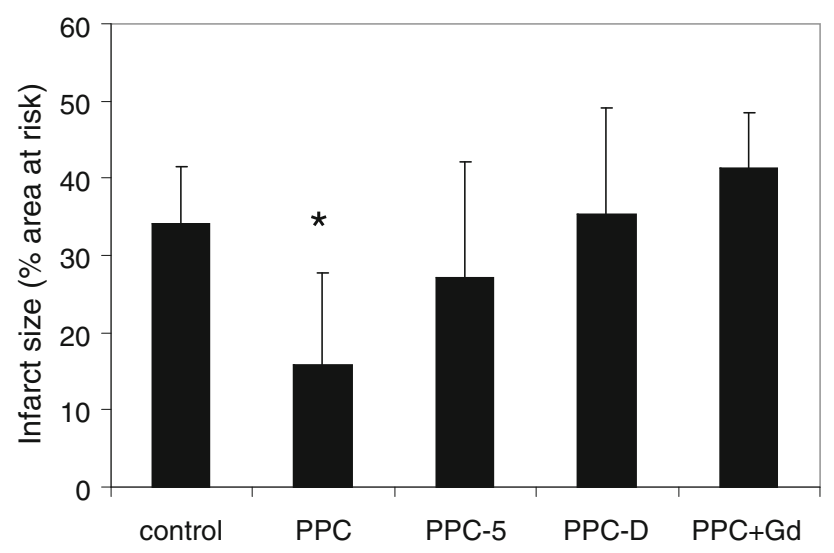

Fig. 7 Infarct size in pig hearts in vivo (expressed as percentage of the area at risk), comparing control hearts $(n=5)$ with those subjected to ten cycles of RV pacing (PPC, $n=6$ ), five cycles of RV pacing (PPC-5, $n=5$ ), ten cycles of RV pacing, but starting 5 min after reperfusion (PPC-D, $n=5$ ) and ten cycles of RV pacing in combination with administration of gadolinium (PPC-Gd, $n=5$ ). Values are mean $\pm \mathrm{SD} . * P<0.05$, as compared to control

the ten pacing cycles as late as $5 \mathrm{~min}$ after onset of reperfusion abrogated the protective effect of PPC completely. Similarly, administration of the SAC blocker gadolinium completely abrogated the infarct size reduction provided by PPC.

In the entire group of 26 animals, 8 experienced ventricular fibrillation (VF) during the occlusion period and 6 in the early reperfusion phase. With respect to the latter, this concerned 2/5 animals in the control group, 2/6 in the $\mathrm{PPC}, 0 / 5$ in the PPC $+\mathrm{Gd}, 1 / 5$ in the PPC-5 and $1 / 5$ in the PPC-D group. In all those cases, VF occurred during the first 2 min after the start of reperfusion. VF occurred in the absence of pacing (control group) as well as before onset of the pacing protocol (PPC-D group). Also, the two cases of VF in the PPC group occurred immediately on reperfusion, before starting the pacing protocol. All these animals were successfully defibrillated and their data were included in the analysis.

\section{Discussion}

This study demonstrates that PPC achieves a maintained reduction in infarct size, which will be of crucial importance for potential application in a clinical setting. The data from the present study provide further evidence that abnormal mechanical loading of the myocardium is a trigger for the PPC-induced protection. SACs and microtubules appear to play a role in transduction of the stretch signal to more downstream located pathways, which appear to be similar in PPC and other modes of postconditioning.
Long-term protection by PPC

The demonstration that PPC induces maintained reduction in infarct size strongly indicates that PPC does not delay, but actually diminishes, myocardial injury over the long term. Long-term infarct size reduction has also been shown in experimental [19] and clinical studies on IPoC [37]. The present study, therefore, provides support for a potentially clinically relevant, maintained protection by PPC in patients. The smaller infarct size in the PPC animals was associated with a lack of the transient decrease in fractional shortening after 2 weeks of myocardial infarction, as observed in the control animals. The absence of more pronounced effect of the protection on cardiac geometries may be due to the relatively small infarcts $(\sim 20 \%$ of $\mathrm{LV}$ mass) and the ability to compensate for the loss of function, as evidenced from the recovery of fractional shortening in the control group. As reviewed by Miura and Miki [18], also in patients a significant effect on echocardiographic parameters can only be expected when a considerable reduction in infarct size is achieved.

\section{Mechanisms of PPC}

The lack of effect of the adenosine receptor blocker 8-SPT on PPC provides further evidence that ischemia is not the trigger for PPC. This evidence corroborates findings from our previous study that ventricular pacing does not change myocardial blood flow or lactate release during normal conditions or during early reperfusion [39]. Therefore, also other mechanisms such as maintained acidosis [29] are not expected to play a role. Because PPC was applied while applying immediate and full reperfusion, the protection afforded by PPC cannot be related to gradual reperfusion and is a strong argument for postconditioning as a separate entity [10].

Ventricular pacing markedly alters the myocardial contraction pattern, leading to considerable regional differences in mechanical load in vivo and in the isolated working heart model [26, 27, 39, 40]. Since in our isolated heart preparation many hemodynamic factors (heart rate, global preload, global afterload) were kept constant, the intermittent changes in regional mechanical loading are a likely candidate to be the trigger for PPC. The abnormal mechanics during ventricular pacing is characterized by early systolic stretch in late-activated regions (those remote from the pacing site). The idea that such stretch could elicit protection is supported by the observation that intermittently increasing global preload is also protective and that a protective effect is absent when performing $\mathrm{BiV}$ pacing, which is associated with less abnormal contraction patterns than LV pacing [43]. These findings indicate that alterations in workload of the myocardium, and not the artificial 
electrical stimulation, have a protective effect. The lack of protection in the $\mathrm{BiV}$ pacing group excludes the possibility that the electrical stimulation may activate autonomic cardiac nerves, thereby causing protection [2].

This study is also the first to show that increased global preload induces postconditioning, but earlier studies have shown this for preconditioning $[8,22]$. The abolishment of PPC-induced protection by gadolinium indicates that SACs may be involved in the transduction of the protective stimulus. Gadolinium is a rather non-specific blocker of SACs. Nevertheless, it has been shown to block cardioprotection evoked by volume infusion before the index ischemia (stretch-induced preconditioning) $[8,22]$. This finding supports the idea that PPC-induced protection depends on myocardial stretch as a trigger or mediator. This trigger should be applied in at least $>5$ cycles, whereas the duration of each cycle is less important (Figs. 6, 7). This observation indicates that the myocardium may adapt quickly to the stretch stimulus.

The aforementioned stretch hypothesis is also supported by the finding that the microtubule-disrupting agent colchicine abolished PPC-induced reduction in infarct size. Microtubules form a network of fibers between sarcolemma and nucleus (which is deformed when the myocyte is deformed [21]) and play a key role in mechanotransduction.

Stretching of myocytes leads to release of angiotensin, which is known to induce a hypertrophic response in the myocyte through autocrine/paracrine effects. However, the observation that the AT-1 blocker candesartan does not reduce protection by PPC indicates that this mechanism does not contribute importantly to the protective effect of PPC.

\section{Downstream pathways}

In the present study, we used various pharmacological blockers of pathways to explore their involvement in PPC. This is a limitation of the study, because the blockers may not be specific. Ultimate proof of a role of a pathway is given by direct demonstration of activation of the pathway, for example using Western blot analysis for degree of phosphorylation. The main message from the present study is not the involvement of one specific pathway in PPC, but rather that PPC reacts in a similar fashion to several blockers of classical downstream pathways of protection. Therefore, these data indicate that, while upstream IPoC and PPC show clear differences (no involvement of adenosine receptors in PPC), their downstream pathways may be more similar.

PKC activation, shown in this study to be involved in PPC, is also known as an important intermediate step in protocols for IPoC [42]. In ischemic preconditioning, it has been shown that PKC is most likely downstream of the adenosine receptor, because the combination of PKC activation and adenosine receptor blockade was protective, whereas adenosine receptor activation in combination with PKC blockade was not [13].

The Pi3K-Akt is another well-known pathway in cardioprotection and is also referred to as the RISK pathway. This pathway is implemented in IPoC in rats and rabbits [44]. However, its role in pigs is debated [30, 32]. In this species, IPoC did not induce additional activation of RISK or protection [32]. The abolishment of PPC-induced protection by wortmannin suggests involvement of the RISK pathway in PPC. It should be recognized that a protectionabolishing effect of wortmannin is not a direct proof of the involvement of the RISK pathway. However, in the study on involvement of the RISK pathway in pigs, a lack of effect of wortmannin was associated with a lack of direct involvement of the RISK pathway [32], whereas in a study on the postconditioning effect of levosimendan the increased phosphorylation of Akt and GSK-3b was reversed completely in hearts that received wortmannin in addition to levosimendan [12]. Therefore, wortmannin may provide a fairly reliable prediction of involvement of the RISK pathway in PPC in our setting of isolated rabbit hearts. In the same experimental setting and species, Yang et al. [44] also showed that wortmannin abolished protection by IPoC. So at least in the rabbit heart, PPC appears to share the RISK pathway with IPoC.

The fact that we also observed protection by PPC in pigs may suggest that (also) other pathways are involved. A good candidate in this respect is the tumor necrosis factor alpha $(\mathrm{TNF} \alpha)$ pathway. Even brief periods of mechanical stretch can induce rapid phosphorylation of JAK, STAT and glycoprotein 130 [23]. TNF $\alpha$-induced cardioprotection does not rely on AKT and ERK1/2 [11] and TNF $\alpha$ is also cardioprotective in pigs. [31].

The abolishment of the cardioprotective effect of PPC by the selective mitoK $_{\text {ATP }}$ channel blocker 5HD indicates that opening of this channel is important in the cardioprotection provided by PPC, similar to that provided by IPoC. In ischemic preconditioning and postconditioning, the mitoK $\mathrm{K}_{\mathrm{ATP}}$ channel has been assigned both a role as trigger and as mediator $[41,47]$. If the mitoK $_{\mathrm{ATP}}$ would act as mediator in PPC, it may be activated by PKC and Pi3K. A more direct involvement of mitoK $_{\text {ATP }}$ in PPC could be imagined to initiate from metabolic signals originating from the mitochondria, the oxygen consumption of which presumably change during each onset and end of a cycle of ventricular pacing due to changes in mechanical load [27].

Another downstream target of PI3K in PPC could be HIF $1 \alpha$. In a recent study, HIF $1 \alpha$ was shown to be related to protection afforded by IPoC [49]. Moreover, increased wall 
stress has been shown to increase HIF1 $\alpha$, using a SACPI3K pathway [15].

Data from the pig heart studies demonstrate that PPC should be applied within the first $5 \mathrm{~min}$ after reperfusion. A similar time window is known to exist for IPoC in rabbit and human hearts [33].

Our finding that protection is achieved by ten, and not by five, cycles of pacing in the pig heart are in line with the data from Iliodromitis et al. [14], who found that protection was achieved by eight and not four 30-s cycles of IPoC. It is remarkable, however, that in the current study neither application of five PPC cycles immediately after reperfusion nor ten PPC cycles starting $5 \mathrm{~min}$ after reperfusion is effective, but that adding five cycles of pacing after the first five cycles at the onset of reperfusion is required to provide protection. This implies that the protective signaling processes are not completed within 5 min of reperfusion and need to be amplified by the second five pacing cycles.

\section{Potential clinical application}

PPC has the potential to be applied clinically. First of all, the pacing hardware (pacing leads and pacemakers) has already been developed and used in patients for many years. Theoretically, PPC could be used in heart transplantation cardiac surgery and percutaneous coronary intervention conditions where beneficial effects of IPoC have been shown [16, 17, 34]. Moreover, a postconditioning algorithm could be implemented in implantable devices, such as pacemakers and implantable cardioverter defibrillators. The latter option is unique for PPC.

Ventricular pacing may have the advantage over "classical" IPoC, in that it does not require mechanical interaction with the already vulnerable coronary arterial wall. PPC may be more complicated to "administer" than drugs, but pacing and its side effects may be more easily controllable, especially in the setting of percutaneous coronary intervention.

An important issue is whether ventricular pacing in the early reperfusion phase is proarrhythmic. During the early reperfusion phase, the heart is susceptible to arrhythmia and ectopic stimulation protocols are being used as provocation tests for arrhythmias. However, in the present study no evidence was found for a pro-arrhythmic effect. This is notable, because pigs are known to be quite sensitive to arrhythmias during ischemia/reperfusion experiments. These findings may be explained by the use of the VVI pacing mode, which inhibits stimulation when a spontaneous activation of the myocardium is sensed. Moreover, pacing was performed at a regular rate, only slightly above natural sinus rhythm. This is in contrast to arrhythmia provocation tests, where stimulation is performed at a high rate and/or at variable intervals. Clearly, further investigation is required to assure the safety of PPC in a clinical setting.

PPC may also be useful as an additive trigger for postconditioning, in conjunction with other postconditioning approaches. It has been shown for example that the combination of drugs and ischemic pre-conditioning has additive effects [20]. Such additive effect may be of particular interest to patients with compromised hearts, who are known to be more susceptible to myocardial damage and less responsive to preconditioning and postconditioning stimuli [6, 14, 36, 38, 50]. A study in ob/ob mice showed reduced cardioprotection by IPoC, which was related to the lack of phosphorylation of signaling molecules [5]. Application of modes of postconditioning with different triggering mechanisms may be helpful in achieving sufficient levels of protection.

Finally, the finding that protection by PPC is independent of the adenosine receptor may make this protection insensitive to elevated plasma levels of caffeine. After all, it has been shown that caffeine blocks the adenosine receptor and that it also abolishes the protective effect on ischemia/reperfusion damage by ischemic preconditioning [28] and by statins [46]. Because caffeine is frequently consumed and has a half-life of many hours, many patients entering the hospital with a myocardial infarction may have impaired potential to be protected by interventions, such as IPoC and drugs like statins and bradykinin. PPC could be a promising alternative, since it is independent of adenosine receptors, as shown by the lack of effect of 8-SPT, which is a caffeine derivate.

\section{Conclusion}

The present study shows that PPC, a recently discovered mode of protection, results in maintained reduction of infarct size and that abnormal mechanical load is the trigger of the PPC-induced protection, presumably transduced by stretch-activated channels. PPC shares downstream signaling pathways with other modes of cardioprotection, such as PKC and PI3K activation. Interestingly, however, adenosine and angiotensin receptors are not involved in PPC-induced protection. Therefore, PPC may be useful, because its effect is not hampered by caffeine and other G-protein signaling blocking substances.

Acknowledgments This study was financially supported by Boston Scientific CRM, St. Paul USA.

Open Access This article is distributed under the terms of the Creative Commons Attribution Noncommercial License which permits any noncommercial use, distribution, and reproduction in any medium, provided the original author(s) and source are credited. 


\section{References}

1. Argaud L, Gateau-Roesch O, Raisky O, Loufouat J, Robert D, Ovize M (2005) Postconditioning inhibits mitochondrial permeability transition. Circulation 111:194-197

2. Banerjee A, Locke-Winter C, Rogers KB, Mitchell MB, Brew EC, Cairns CB, Bensard DD, Harken AH (1993) Preconditioning against myocardial dysfunction after ischemia and reperfusion by an alpha 1-adrenergic mechanism. Circ Res 73:656-670

3. Blombery PA, Korner PI (1982) Role of aortic and carotid sinus baroreceptors on Valsalva-like vasoconstrictor and heart rate reflexes in the conscious rabbit. J Auton Nerv Syst 5:303-315

4. Bode F, Katchman A, Woosley RL, Franz MR (2000) Gadolinium decreases stretch-induced vulnerability to atrial fibrillation. Circulation 101:2200-2205

5. Bouhidel O, Pons S, Souktani R, Zini R, Berdeaux A, Ghaleh B (2008) Myocardial ischemic postconditioning against ischemiareperfusion is impaired in ob/ob mice. Am J Physiol Heart Circ Physiol 295:H1580-H1586

6. Ferdinandy P, Szilvassy Z, Baxter GF (1998) Adaptation to myocardial stress in disease states: is preconditioning a healthy heart phenomenon? Review. Trends Pharmacol Sci 19:223-229

7. Fryer RM, Hsu AK, Gross GJ (2001) Mitochondrial K(ATP) channel opening is important during index ischemia and following myocardial reperfusion in ischemic preconditioned rat hearts. J Mol Cell Cardiol 33:831-834

8. Gysembergh A, Margonari H, Loufoua J, Ovize A, Andre-Fouet X, Minaire Y, Ovize M (1998) Stretch-induced protection shares a common mechanism with ischemic preconditioning in rabbit heart. Am J Physiol 274:H955-H964

9. Hansen D, Borganelli M, Stacy GPJ, Taylor LK (1991) Dosedependent inhibition of stretch-induced arrhythmias by gadolinium in isolated canine ventricles. Evidence for a unique mode of antiarrhythmic action. Circ Res 69:820-831

10. Heusch G (2004) Postconditioning: old wine in a new bottle? J Am Coll Cardiol 44:1111-1112

11. Heusch G, Boengler K, Schulz R (2008) Cardioprotection: nitric oxide, protein kinases, and mitochondria. Circulation 118:19151919

12. Hoenisch A, Theuring N, Ebner B, Wagner C, Strasser RH, Weinbrenner C (2010) Postconditioning with levosimendan reduces the infarct size involving the PI3K pathway and KATPchannel activation but is independent of PDE-III inhibition. Basic Res Cardiol 105:155-167

13. Iliodromitis EK, Miki T, Liu GS, Downey JM, Cohen MV, Kremastinos DT (1998) The PKC activator PMA preconditions rabbit heart in the presence of adenosine receptor blockade: is $5^{\prime}$ nucleotidase important? J Mol Cell Cardiol 30:2201-2211

14. Iliodromitis EK, Zoga A, Vrettou A, Andreadou I, Paraskevaidis IA, Kaklamanis L, Kremastinos DT (2006) The effectiveness of postconditioning and preconditioning on infarct size in hypercholesterolemic and normal anesthetized rabbits. Atherosclerosis 188:356-362

15. Kim CH, Cho YS, Chun YS, Park JW, Kim MS (2002) Early expression of myocardial HIF-1alpha in response to mechanical stresses: regulation by stretch-activated channels and the phosphatidylinositol 3-kinase signaling pathway. Circ Res 90:E25E33

16. Lauzier B, Sicard P, Bouchot O, Delemasure S, Menetrier F, Moreau D, Vergely C, Rochette L (2007) After four hours of cold ischemia and cardioplegic protocol, the heart can still be rescued with postconditioning. Transplantation 84:1382-1383

17. Luo W, Li B, Chen R, Huang R, Lin G (2008) Effect of ischemic postconditioning in adult valve surgery. Eur J Cardiothorac Surg 33:203-208
18. Miura T, Miki T (2008) Limitation of myocardial infarct size in the clinical setting: current status and challenges in translating animal experiments into clinical therapy. Basic Res Cardiol 103:501-513

19. Mykytenko J, Kerendi F, Reeves JG, Kin H, Zatta AJ, Jiang R, Guyton RA, Vinten-Johansen J, Zhao ZQ (2007) Long-term inhibition of myocardial infarction by postconditioning during reperfusion. Basic Res Cardiol 102:90-100

20. Ninomiya $\mathrm{H}$, Otani $\mathrm{H}$, Lu K, Uchiyama T, Kido M, Imamura $\mathrm{H}$ (2002) Enhanced IPC by activation of pertussis toxin-sensitive and -insensitive $G$ protein-coupled purinoceptors. Am J Physiol 282:H1933-H1943

21. Nishimura S, Nagai S, Katoh M, Yamashita H, Saeki Y, Okada J, Hisada T, Nagai R, Sugiura S (2006) Microtubules modulate the stiffness of cardiomyocytes against shear stress. Circ Res 98:81-87

22. Ovize M, Kloner RA, Przyklenk K (1994) Stretch preconditions canine myocardium. Am J Physiol 266:H137-H146

23. Pan J, Fukuda K, Saito M, Matsuzaki J, Kodama H, Sano M, Takahashi T, Kato T, Ogawa S (1999) Mechanical stretch activates the JAK/STAT pathway in rat cardiomyocytes. Circ Res 84:1127-1136

24. Patel VC, Yellon DM, Singh KJ, Neild GH, Woolfson RG (1993) Inhibition of nitric oxide limits infarct size in the in situ rabbit heart. Biochem Biophys Res Commun 194:234-238

25. Piper HM, Schafer AC (2004) The first minutes of reperfusion: a window of opportunity for cardioprotection. Cardiovasc Res 61:365-371

26. Prinzen FW, Augustijn CH, Arts T, Allessie MA, Reneman RS (1990) Redistribution of myocardial fiber strain and blood flow by asynchronous activation. Am J Physiol 259:H300-H308

27. Prinzen FW, Hunter WC, Wyman BT, McVeigh ER (1999) Mapping of regional myocardial strain and work during ventricular pacing: experimental study using Magnetic Resonance Imaging tagging. J Am Coll Cardiol 33:1735-1742

28. Riksen NP, Zhou Z, Oyen WJ, Jaspers R, Ramakers BP, Brouwer RM, Boerman OC, Steinmetz N, Smits P, Rongen GA (2006) Caffeine prevents protection in two human models of ischemic preconditioning. J Am Coll Cardiol 48:700-707

29. Rodríguez-Sinovas A, Cabestrero A, García del Blanco B, Inserte J, García A, García-Dorado D (2009) Intracoronary acid infusion as an alternative to ischemic postconditioning in pigs. Basic Res Cardiol 104:761-771

30. Schwartz LM, Welch TS, Crago MS (2002) Cardioprotection by multiple preconditioning cycles does not require mitochondrial $\mathrm{K}(\mathrm{ATP})$ channels in pigs. Am J Physiol Heart Circ Physiol 283:H1538-H1544

31. Skyschally A, Gres P, Hoffmann S, Haude M, Erbel R, Schulz R, Heusch G (2007) Bidirectional role of tumor necrosis factoralpha in coronary microembolization: progressive contractile dysfunction versus delayed protection against infarction. Circ Res 100:140-146

32. Skyschally A, van Caster P, Boengler K, Gres P, Musiolik J, Schilawa D, Schulz R, Heusch G (2009) Ischemic postconditioning in pigs: no causal role for RISK activation. Circ Res 104:15-18

33. Skyschally A, van Caster P, Iliodromitis EK, Schulz R, Kremastinos DT, Heusch G (2009) Ischemic postconditioning: experimental models and protocol algorithms. Basic Res Cardiol 104:469-483

34. Staat P, Rioufol G, Piot C, Cottin Y, Cung TT, L'Huillier I, Aupetit JF, Bonnefoy E, Finet G, Andre-Fouet X, Ovize M (2005) Postconditioning the human heart. Circulation 112:21432148

35. Takano H, Tang XL, Bolli R (2000) Differential role of K(ATP) channels in late preconditioning against myocardial stunning and 
infarction in rabbits. Am $\mathrm{J}$ Physiol Heart Circ Physiol 279:H2350-H2359

36. Tang XL, Takano H, Xuan YT, Sato H, Kodani E, Dawn B, Zhu Y, Shirk G, Wu WJ, Bolli R (2005) Hypercholesterolemia abrogates late preconditioning via a tetrahydrobiopterin-dependent mechanism in conscious rabbits. Circulation 112:2149-2156

37. Thibault H, Piot C, Staat P, Bontemps L, Sportouch C, Rioufol G, Cung TT, Bonnefoy E, Angoulvant D, Aupetit JF, Finet G, Andre-Fouet X, Macia C, Raczka F, Rossi R, Itti R, Kirkorian G, Derumeaux G, Ovize M (2008) Long-term benefit of postconditioning. Circulation 117:1037-1044

38. Tosaki A, Engelman DT, Engelman RM, Das DK (1996) The evolution of diabetic response to ischemia/reperfusion and preconditioning in isolated working rat hearts. Cardiovasc Res 31:526-536

39. Vanagt WY, Cornelussen RN, Baynham TC, Van Hunnik A, Poulina QP, Babiker F, Spinelli J, Delhaas T, Prinzen FW (2007) Pacing-induced dyssynchrony during early reperfusion reduces infarct size. J Am Coll Cardiol 49:1813-1819

40. Vanagt WY, Cornelussen RN, Poulina QP, Blaauw E, Vernooy K, Cleutjens JP, van Bilsen M, Delhaas T, Prinzen FW (2006) Pacing-induced dys-synchrony preconditions rabbit myocardium against ischemia/reperfusion injury. Circulation 114:I264-I269

41. Vinten-Johansen J, Zhao Z-Q, Jiang R, Zatta AJ, Dobson GP (2007) Preconditioning and postconditioning: innate cardioprotection from ischemia-reperfusion injury. J Appl Physiol 103:1441-1448

42. Vinten-Johansen J, Zhao ZQ, Zatta AJ, Kin H, Halkos ME, Kerendi F (2005) Postconditioning - a new link in nature's armor against myocardial ischemia-reperfusion injury. Basic Res Cardiol 100:295-310

43. Wyman BT, Hunter WC, Prinzen FW, Faris OP, McVeigh ER (2002) Effects of single- and biventricular pacing on temporal and spatial dynamics of ventricular contraction. Am J Physiol Heart Circ Physiol 282:H372-H379

44. Yang XM, Philipp S, Downey JM, Cohen MV (2005) Postconditioning's protection is not dependent on circulating blood factors or cells but involves adenosine receptors and requires PI3kinase and guanylyl cyclase activation. Basic Res Cardiol 100:57-63

45. Yao Z, Gross GJ (1994) A comparison of adenosine-induced cardioprotection and ischemic preconditioning in dogs. Efficacy, time course, and role of KATP channels. Circulation 89:12291236

46. Ye Y, Abu Said G, Lin Y, Manickavasagam S, Hughes M, McAdoo D et al (2008) Caffeinated coffee blunts the myocardial protective effects of statins against ischemia-reperfusion injury in the rat. Cardiovasc Drugs Ther 22:275-282

47. Yellon DM, Downey JM (2003) Preconditioning the myocardium: from cellular physiology to clinical cardiology. Physiol Rev 83:1113-1151

48. Zhang SJ, Yang XM, Liu GS, Cohen MV, Pemberton K, Downey JM (2003) CGX-1051, a peptide from Conus snail venom, attenuates infarction in rabbit hearts when administered at reperfusion. J Cardiovasc Pharmacol 42:764-771

49. Zhao HX, Wang XL, Wang YH, Wu Y, Li XY, Lv XP, Zhao ZQ, Zhao RR, Liu HR (2010) Attenuation of myocardial injury by postconditioning: role of hypoxia inducible factor-1alpha. Basic Res Cardiol 105:109-118

50. Zhao JL, Yang YJ, You SJ, Cui CJ, Gao RL (2006) Different effects of postconditioning on myocardial no-reflow in the normal and hypercholesterolemic mini-swines. Microvasc Res 73:137142

51. Zhao Z-Q, Corvera JS, Halkos ME, Kerendi F, Wang N-P, Guyton RA, Vinten-Johansen J (2003) Inhibition of myocardial injury by ischemic postconditioning during reperfusion: comparison with ischemic preconditioning. Am J Physiol Heart Circ Physiol 285:H579-H588

52. Zhao Z-Q, Nakamura M, Wang N-P, Velez DA, Hewan-Lowe KO, Guyton RA, Vinten-Johansen J (2000) Dynamic progression of contractile and endothelial dysfunction and infarct extension in the late phase of reperfusion. J Surg Res 94:1-12 